\title{
Luminescence properties of lanthanide doped alkaline earth chlorides under (V)UV and X-ray excitation
}

\author{
Aleksander Zych*, Anke Leferink op Reinink, Koen van der Eerden, Celso de Mello Donegá, \\ Andries Meijerink
}

CMI, Debye Institute for NanoMaterials Science, Utrecht University, Princetonplein 1, 3508TA Utrecht, The Netherlands

\section{A R T I C L E I N F O}

Article history:

Received 10 November 2010

Received in revised form 14 January 2011

Accepted 19 January 2011

Available online 26 January 2011

\section{Keywords:}

Rare earth alloys and compounds

Synchrotron radiation

Luminescence

Insulators

\begin{abstract}
A B S T R A C T
The photoluminescence and radioluminescence of $\mathrm{Ce}^{3+}, \mathrm{Pr}^{3+}$ and $\mathrm{Nd}^{3+}$ in $\mathrm{SrCl}_{2}$ and $\mathrm{BaCl}_{2}$ are reported and discussed in relation to application as a (fast) scintillator material. The $\mathrm{Ce}^{3+}$ doped materials exhibit the typical fast cerium $d-f$ emission $\left(358,382 \mathrm{~nm}\right.$ in $\mathrm{SrCl}_{2}$ and $350,375 \mathrm{~nm} \mathrm{in} \mathrm{BaCl}_{2}$ ) both under synchrotron and ionizing radiation excitations. A weak afterglow is observed. For $\operatorname{Pr}^{3+}$ a very fast $d-f$ emission is observed in $\mathrm{SrCl}_{2}(250,263,300$ and $328 \mathrm{~nm}, \tau=13 \mathrm{~ns})$ and $\mathrm{BaCl}_{2}(260,288$ and $315 \mathrm{~nm}, \tau=10 \mathrm{~ns})$, but only under direct $f-d$ excitation. No $d-f$ luminescence is observed for $\mathrm{Pr}^{3+}$ under X-ray excitation. The absence of $d-f$ emission is explained by the energy of the host lattice emission which is too low for energy transfer to the high energy $f d$ states of $\mathrm{Pr}^{3+}\left(230 \mathrm{~nm}\right.$ in $\mathrm{SrCl}_{2}$ and $235 \mathrm{~nm}$ in $\left.\mathrm{BaCl}_{2}\right)$. The neodymium doped chlorides do not show $d-f$ luminescence.
\end{abstract}

(C) 2011 Elsevier B.V. All rights reserved.

\section{Introduction}

Inorganic materials doped with lanthanide ions are well-known for their efficient luminescence related to the unique optical properties of the lanthanides originating from the partially filled $4 f$ shell. As a result, lanthanide doped materials are widely used in applications where efficient emission of light is required, for example in lighting (fluorescent tubes, white light LEDs), displays and medical imaging. In the field of medical imaging there has been great interest in the past decades in the development of new scintillation materials for the conversion of high energy X-rays or gamma-rays into light. Mostly, scintillators are inorganic crystalline materials (e.g. halides, oxides, silicates, aluminates, tungstates), which emit short flashes of light in the UV-vis spectral range upon high energy radiation or particle irradiation. There are some stoichiometric scintillators known but most scintillators rely on emission from luminescent ions (activators) doped into the host lattice. The high energy radiation is deposited in the host lattice where it leads to the creation of electron-hole $(\mathrm{e}-\mathrm{h})$ pairs which recombine on or nearby the activator ion giving rise to efficient emission from the activator. Scintillators are of increasing interest due to a rise in the need for high energy radiation detectors, e.g. in high energy physics, space exploration, home land security and medical imaging. In the field of medical imaging scintillators are applied in positron emission tomography (PET), computed tomography (CT) and other

\footnotetext{
* Corresponding author. Tel.: +31 30 2532203; fax: +31 302532403.

E-mail addresses: a.k.zych@uu.nl, alek_zych@yahoo.com (A. Zych).
}

$\mathrm{X}$-ray imaging techniques and single photon emission computed tomography (SPECT). In the more advanced medical imaging techniques (PET, CT) better scintillators will lead to an increase in the precision of measurements to diagnose cancer earlier and to pinpoint the location of the tumor tissue in the patient's body, as well as decrease the exposure of the patients to the harmful ionizing radiation used in these examinations. The price of the scintillator materials used in these medical imaging machines is an important factor. PET is the technique with the most demanding requirements on the scintillators $[1,2]$. The emission must not only be efficient (preferably the scintillator material should generate at least 20,000 photons/MeV of incoming radiation) but also very fast, with the emission lifetime shorter than $40 \mathrm{~ns}$, since the technique relies on the co-incident detection of gamma-rays emitted at an angle of $180^{\circ}$ upon positron-electron annihilation.

Efficient and fast emission is achieved by incorporation of trivalent $\mathrm{Ce}^{3+}$ ions $\left(4 f^{1}\right)$ in an inorganic host lattice. The $5 d-4 f(d-f)$ emission of $\mathrm{Ce}^{3+}$ is due to fully allowed (parity and spin) transition with a typical radiative lifetime of $25-70 \mathrm{~ns}$. A wide variety of $\mathrm{Ce}^{3+}$ doped materials has been investigated for application in PET scanners. At the same time, it has been realized that an even faster response leads to a better timing resolution and better discrimination between random and real events, giving a higher spatial resolution. New materials with faster response time can use the $d-f$ emission of $\mathrm{Pr}^{3+}\left(4 f^{2}\right)$ or $\mathrm{Nd}^{3+}\left(4 f^{3}\right)$. The $d-f$ emission from $\mathrm{Pr}^{3+}$ and $\mathrm{Nd}^{3+}$ is at higher energies which leads to faster radiative decay based on Fermi's golden rule [3,4].

Here we report the spectroscopic properties of $\mathrm{Ce}^{3+}, \mathrm{Pr}^{3+}$ and $\mathrm{Nd}^{3+}$ in chloride host lattices. The choice for a chloride 
host is motivated by the recently reported high photon yields $\left(>80,000\right.$ photons/MeV) for $\mathrm{Ce}^{3+}$ in $\mathrm{LnX}_{3}(\mathrm{Ln}=\mathrm{La}, \mathrm{Lu} ; \mathrm{X}=\mathrm{Cl}, \mathrm{Br}, \mathrm{I})$ [5-8]. Possibly, incorporation of $\mathrm{Pr}^{3+}$ in a chloride host leads to a combination of a faster decay and high photon yield. Alkaline earth halides are known to show fast emission when doped with trivalent cerium ions [9-16]. Previous work on $\mathrm{Ce}^{3+}$ doped into alkaline earth chlorides has revealed $\mathrm{Ce}^{3+} d-f$ emission around $360 \mathrm{~nm}$ and $390 \mathrm{~nm}\left(\mathrm{SrCl}_{2}\right)$ and $350 \mathrm{~nm}$ and $375 \mathrm{~nm}\left(\mathrm{BaCl}_{2}\right)$, while the lowest $f-d$ excitation bands are observed around $320 \mathrm{~nm}$ and $335 \mathrm{~nm}$ in strontium and barium chlorides, respectively [10-16]. No reports have been found in the literature on the luminescence of $\operatorname{Pr}^{3+}$ and $\mathrm{Nd}^{3+}$ in $\mathrm{SrCl}_{2}$ or $\mathrm{BaCl}_{2}$. Here we will show that $\mathrm{Pr}^{3+}$ shows fast $d-f$ emission in both $\mathrm{SrCl}_{2}$ and $\mathrm{BaCl}_{2}$. Use of the materials as scintillators is however hampered by the absence of energy transfer from the host lattice to the emitting $f d$ state.

\section{Experimental}

\subsection{Synthesis and characterization}

The materials were synthesized by melting and rapid quenching of the starting materials (anhydrous chlorides) in a glassy carbon crucible in a high frequency furnace, similar to the method reported earlier for sodium chloride [17]. This allows for fast synthesis of simple chemical compositions even in the case of ionic radii and charge mismatch between the dopant and the host lattice cations. The samples obtained are polycrystalline. The mixture of anhydrous chlorides was dried over night at $200^{\circ} \mathrm{C}$ under a constant nitrogen flow. Then the temperature was raised to $300^{\circ} \mathrm{C}$ and maintained as such for $2 \mathrm{~h}$. Subsequently, melting at about $1000^{\circ} \mathrm{C}$ $\left(\mathrm{SrCl}_{2}\right)$ or $1100{ }^{\circ} \mathrm{C}\left(\mathrm{BaCl}_{2}\right)$ took place and after $15 \mathrm{~min}$ the melt was rapidly quenched to room temperature. The samples showed uniform red luminescence (in case of praseodymium doped host lattices) when observed under UV excitation $(254 \mathrm{~nm})$.

The crystal structure of the samples was checked by X-ray powder diffraction (XRD), using a PW 1729 Philips diffractometer, equipped in a $\mathrm{Cu} \mathrm{K}_{\alpha} \mathrm{X}$-ray source $(\lambda=1.5418 \AA$ ) . The XRD patterns of the compounds synthesized have been compared with the theoretical patterns of strontium chloride [18] and barium chloride [19]. Strontium chloride can exist in several different crystal structures. The compounds that have been synthesized are cubic ( $a=b=c=6.98 \AA$ and $\alpha=\beta=\gamma=90^{\circ}$ ), with a density of $3.09 \mathrm{~g} / \mathrm{cm}^{3}$. Barium chloride can also crystallize in various crystal structures depending on the synthesis conditions. The samples that were obtained were orthorhombic $\left(a=7.88 \AA, b=9.42 \AA, c=4.73 \AA\right.$ and $\left.\alpha=\beta=\gamma=90^{\circ}\right)$, with a density of $3.94 \mathrm{~g} / \mathrm{cm}^{3}$. The strontium chloride materials show some additional peaks in the XRD patterns. This is attributed to the hygroscopicity of the material, which results in structural change due to incorporation of water and creation of different hydrates of strontium chloride, possibly during the collection of the XRD pattern.

\subsection{Optical measurements}

Luminescence spectra have been measured using synchrotron radiation at SUPERLUMI station (DESY, Hamburg) which is an excellent station for vacuum UV (VUV) spectroscopy. A more elaborate description of the set-up can be found elsewhere [20]. In short, the VUV part of the synchrotron spectrum is dispersed in an excitation monochromator giving the option to measure high resolution excitation spectra between $\sim 65$ and $335 \mathrm{~nm}$, with a $0.3 \mathrm{~nm}$ resolution. Emission can be coupled into different monochromator/detector combinations, allowing for the measurement of emission between 120 and $1000 \mathrm{~nm}$. The synchrotron spectra have been measured in three time windows (integrated, fast and slow) making it possible to distinguish between fast and slow emissions. Luminescence lifetimes have been recorded with a timing resolution of about $200 \mathrm{ps}$ using time-to-amplitude conversion (TAC). The excitation spectra have been corrected for signal intensity distribution with a spectrum of sodium salicylate. The emission spectra were not corrected for detector response. The samples have been placed in a cold finger cryostat on a copper sample holder.

Emission measurements under soft-X-ray excitation (radioluminescence) have been carried out at room temperature, using the white spectrum of a copper tube operated at $40 \mathrm{kV}$, with the energy in the maximum of the white band of about $28.7 \mathrm{keV}$. Afterglow of the investigated materials has been measured as well, using the same set-up.

Due to the hygroscopic character of the materials investigated, special care was taken to prevent incorporation of water. The samples were stored in sealed plastic bags that were UV transparent. Additionally, the samples have been stored in a desiccator above dry silica beads. Only in case of XRD and synchrotron measurements the samples were taken out of the protective bags. The XRD patterns were recorded under ambient conditions which can explain the observation of small lines related to hydrated phases in some cases.

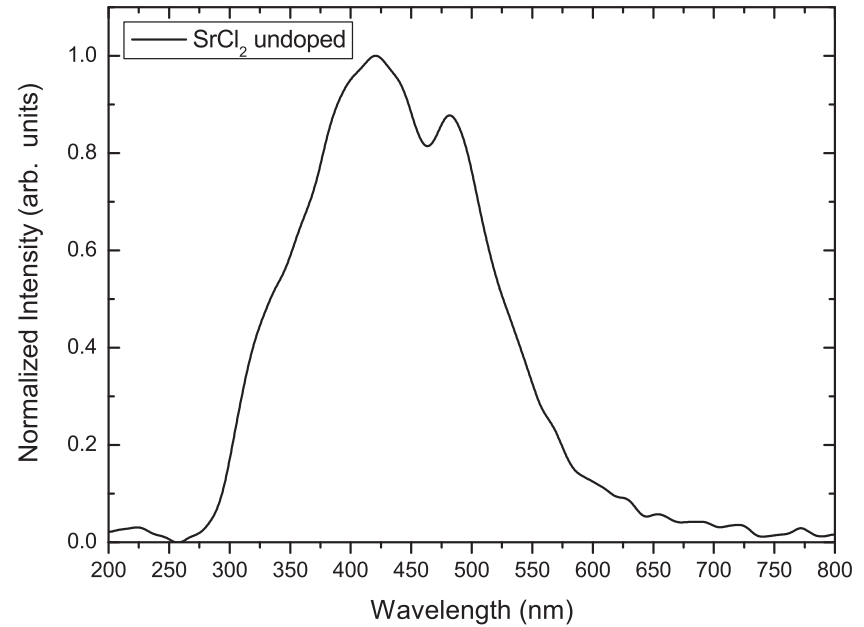

Fig. 1. Emission spectrum of undoped $\mathrm{SrCl}_{2}$ under X-ray irradiation (26.7 keV), at $300 \mathrm{~K}$.

\section{Results and discussion}

\subsection{Luminescence spectroscopy}

\subsubsection{Undoped $\mathrm{SrCl}_{2}$ and $\mathrm{BaCl}_{2}$}

The bandgaps reported in the literature for $\mathrm{SrCl}_{2}$ and $\mathrm{BaCl}_{2}$ differ slightly and have been estimated to be $7.5 \mathrm{eV}(165 \mathrm{~nm})$ and $7.0 \mathrm{eV}(177 \mathrm{~nm}$ ) for strontium chloride and barium chloride, respectively [21]. The estimate is only approximate, since the bandgap depends on the crystal structure, which has not been mentioned in the cited reference [21]. The luminescence of the undoped barium chloride has been extensively studied in the past [22,23]. Two major emission bands are observed with maxima at around $310 \mathrm{~nm}$ and $450 \mathrm{~nm}$. The former has been ascribed to the STE emission and occurs only under excitation in the excitonic absorption peak [22], while the latter has been assigned to the $\mathrm{F}-\mathrm{V}_{\mathrm{k}}$ center recombination [23]. However, no reports on the spectroscopic properties of the undoped $\mathrm{SrCl}_{2}$ host lattice have been found in the literature. In Fig. 1 a typical spectrum of the undoped $\mathrm{SrCl}_{2}$ host lattice under X-ray excitation is presented. Two major emission bands are observed, peaking at around $420 \mathrm{~nm}$ and $483 \mathrm{~nm}$. There is an additional small intensity band present around $225 \mathrm{~nm}$ and a shoulder to the main emission band at $420 \mathrm{~nm}$, which is located at around $328 \mathrm{~nm}$. The exact origin of these bands has not been investigated, though the small intensity band at around $225 \mathrm{~nm}$ might be an STE emission, while the main broad emission band at $420 \mathrm{~nm}$ could be related to the $\mathrm{V}_{\mathrm{k}}$ center radiative recombination, analogues to that observed for $\mathrm{BaCl}_{2}$. Since the crystal structure of both the materials is different, the observed features would be located at different wavelengths. The broadened peak at about $480 \mathrm{~nm}$ might also be a defect of some sort.

\subsection{2. $\mathrm{Ce}^{3+}$ doped $\mathrm{SrCl}_{2}$ and $\mathrm{BaCl}_{2}$}

Strontium chloride has been doped with trivalent cerium ions and the luminescence spectra have been recorded under different excitation wavelengths. A typical emission spectrum is presented in Fig. 2. The black curve corresponds to the emission as observed under a $293 \mathrm{~nm}$ excitation. The emission spectrum shows two broad bands, located at about 358 and $382 \mathrm{~nm}$. The bands are assigned to transitions from the lowest $f d$ state of $\mathrm{Ce}^{3+}$ to the ${ }^{2} \mathrm{~F}_{5 / 2}$ and ${ }^{2} \mathrm{~F}_{7 / 2} 4 f^{1}$ ground state of $\mathrm{Ce}^{3+}$. The observed energy separation between the two bands is about $1755 \mathrm{~cm}^{-1}$, which is close to the expected spin-orbit splitting of $2000 \mathrm{~cm}^{-1}$. It should be noted that in case of strontium chloride the trivalent cerium ions enter the divalent host lattice site. Due to the need of charge compensation 


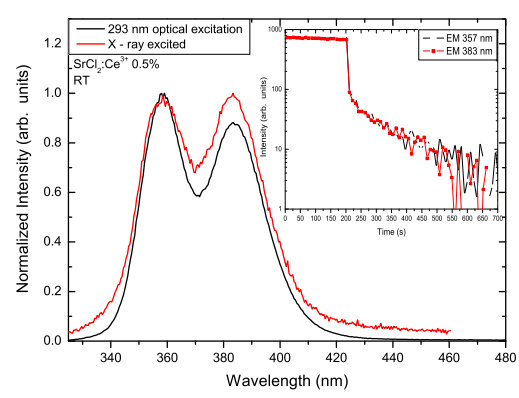

Fig. 2. Emission spectra of $\mathrm{SrCl}_{2}: \mathrm{Ce}^{3+} 0.5 \%$ under UV excitation ( $293 \mathrm{~nm}$, black line) and under X-ray irradiation $(26.7 \mathrm{keV}$, red line), at $300 \mathrm{~K}$. The inset shows the afterglow curves for the $357 \mathrm{~nm}$ and $383 \mathrm{~nm}$ emissions after X-ray irradiation. (For interpretation of the references to color in this figure legend, the reader is referred to the web version of the article.)

multiple lanthanide sites occur in the sample and can also give rise to different emission wavelengths.

The emission lifetime has been measured under direct $f$ - $d$ excitation at $325 \mathrm{~nm}$, monitoring the $340 \mathrm{~nm} \mathrm{Ce}^{3+} d-f$ emission (Fig. 3). For $0.1 \%$ Ce sample a decay time of $26 \mathrm{~ns}$ is measured at $300 \mathrm{~K}$.

In $\mathrm{BaCl}_{2}$ doped with $\mathrm{Ce}^{3+}$ the emission spectrum is similar to that for $\mathrm{SrCl}_{2}: \mathrm{Ce}^{3+}$. There is a slight blue shift of the emission bands, which are located around 350 and $375 \mathrm{~nm}$ for $295 \mathrm{~nm}$ excitation, as shown in Fig. 4 (black curve). The energy separation of the bands is about $1900 \mathrm{~cm}^{-1}$, which corresponds well to the expected separation of $2000 \mathrm{~cm}^{-1}$. Just as for $\mathrm{SrCl}_{2}$, also for $\mathrm{BaCl}_{2}$ differently charge compensated sites may influence the observed splitting in two bands. The results obtained for $\mathrm{BaCl}_{2}: \mathrm{Ce}^{3+}$ are in line with earlier reports in the literature [10-13]. Further comparison with the literature data will follow in the section on radioluminescence. The emission lifetime has been measured under a direct $f$ - $d$ excitation at $275 \mathrm{~nm}$ for $350 \mathrm{~nm}$ emission (Fig. 5). A single exponential fit gives a decay time of $19 \mathrm{~ns}$.

\subsection{3. $\mathrm{Pr}^{3+}$ doped $\mathrm{SrCl}_{2}$ and $\mathrm{BaCl}_{2}$}

Emission spectra were recorded for $\mathrm{SrCl}_{2}: \mathrm{Pr}^{3+}$ under excitation at $165 \mathrm{~nm}$ (the excitonic absorption edge) and $236 \mathrm{~nm}$ (in the lowest energy $f d$ band) at $8 \mathrm{~K}$ (Fig. 6). Under direct excitation in the $f d$ band (Fig. 6A), four emission bands are observed, around 250, 263, 300 and $328 \mathrm{~nm}$, as well as a very broad and low intensity band between 400 and $550 \mathrm{~nm}$ with maxima around 430,460 and $490 \mathrm{~nm}$. The broad band is assigned to a defect luminescence. Due to low reso-

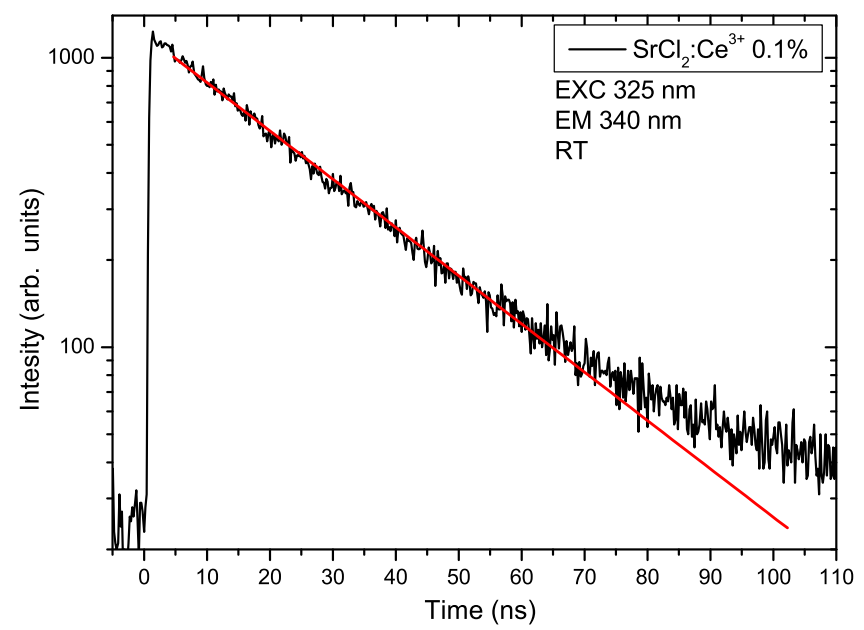

Fig. 3. Luminescence decay curve of the $\mathrm{Ce}^{3+} d-f$ emission ( $340 \mathrm{~nm}$ ) in $\mathrm{SrCl}_{2}: \mathrm{Ce}^{3+}$ $0.1 \%$ under direct excitation in the $f d$ band $(325 \mathrm{~nm})$. The drawn line corresponds to a fit to single exponential decay with $\tau=26 \mathrm{~ns}$.

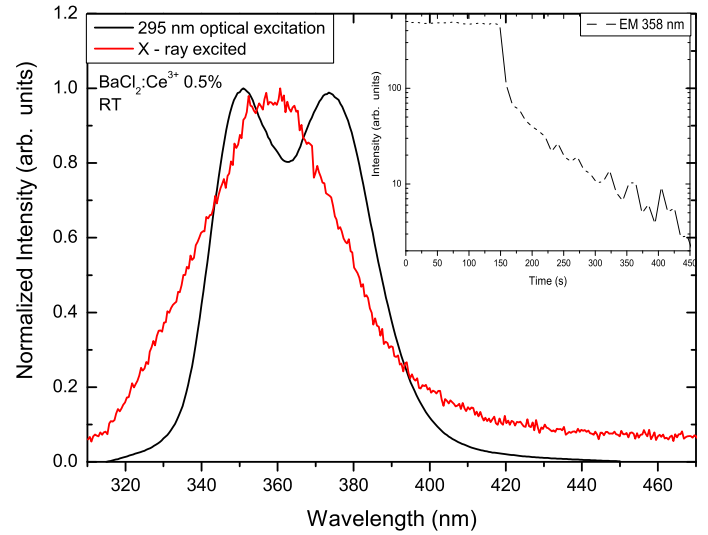

Fig. 4. Emission spectra of $\mathrm{BaCl}_{2}: \mathrm{Ce}^{3+} 0.5 \%$ under UV excitation ( $295 \mathrm{~nm}$, black line) and under X-ray irradiation ( $26.7 \mathrm{keV})$, at $300 \mathrm{~K}$. The inset shows the afterglow curve for the $358 \mathrm{~nm}$ emission after X-ray irradiation. (For interpretation of the references to color in this figure legend, the reader is referred to the web version of the article.)

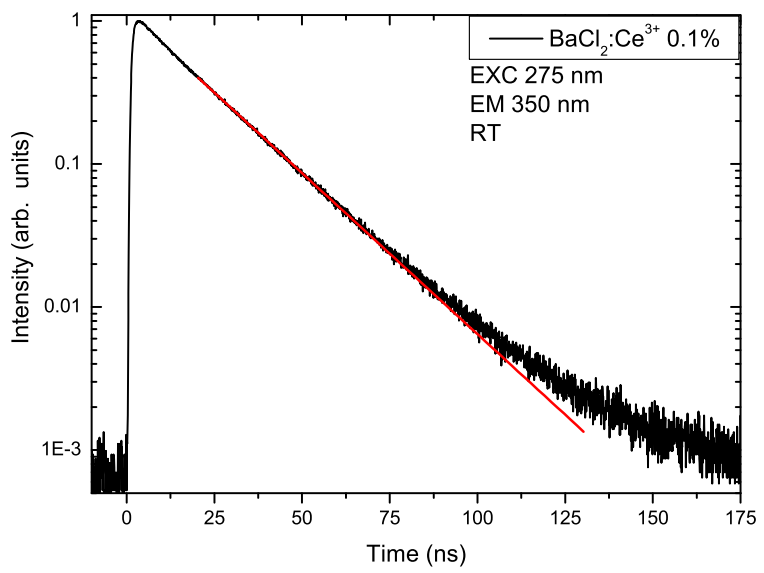

Fig. 5. Luminescence decay curve of the $\mathrm{Ce}^{3+} d-f$ emission $(350 \mathrm{~nm})$ in $\mathrm{BaCl}_{2}: \mathrm{Ce}^{3+}$ $0.1 \%$ under direct excitation in the $f d$ band $(275 \mathrm{~nm})$ at $8 \mathrm{~K}$. The drawn line corresponds to a fit to single exponential decay with $\tau=19 \mathrm{~ns}$.

lution of the emission monochromator used, it is possible that the $490 \mathrm{~nm}$ maximum is in fact the ${ }^{3} \mathrm{P}_{0} \rightarrow{ }^{3} \mathrm{H}_{4}$ transition on $\mathrm{Pr}^{3+}$. The visible emission is stronger in the slow time window, indicating that the decay time is slower than $\sim 100 \mathrm{~ns}$. This is expected for

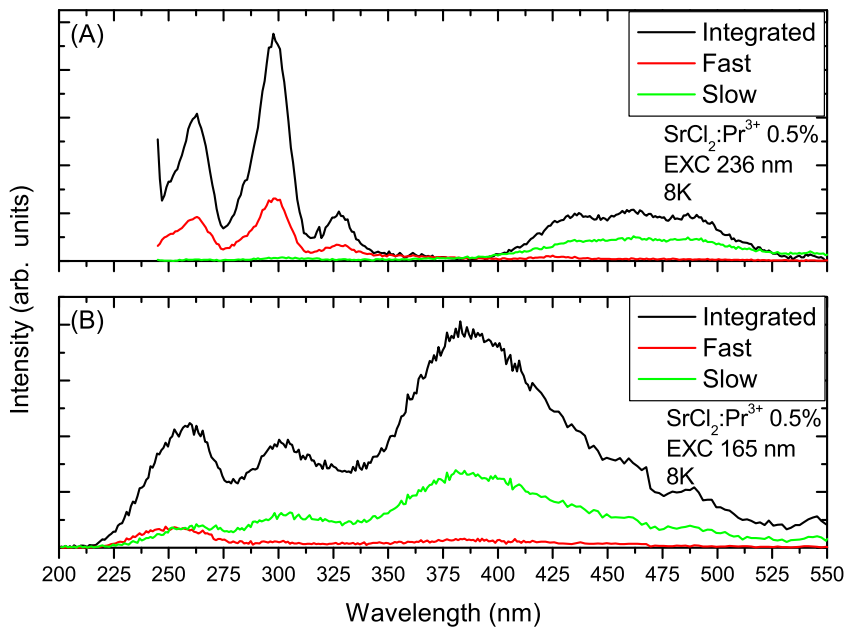

Fig. 6. Emission spectra of $\mathrm{SrCl}_{2}: \operatorname{Pr}^{3+} 0.5 \%$ for excitation into the host lattice at $165 \mathrm{~nm}(\mathrm{~A})$ and direct excitation into the $f d$ band of $\mathrm{Pr}^{3+}$ at $236 \mathrm{~nm}(\mathrm{~B})$. The spectra were recorded in different time windows (fast, slow and integrated, see also Section 2.2) at $8 \mathrm{~K}$. 


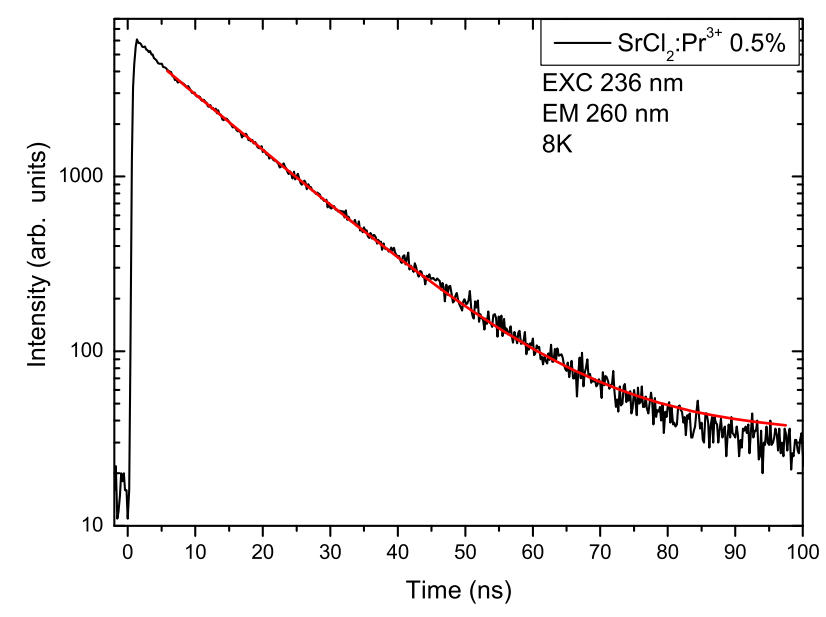

Fig. 7. Luminescence decay curve of the $\operatorname{Pr}^{3+} d-f$ emission (260 nm) in $\mathrm{SrCl}_{2}: \mathrm{Pr}^{3+} 0.5 \%$ under direct excitation in the $f d$ band $(236 \mathrm{~nm})$ at $8 \mathrm{~K}$. The drawn line corresponds to a fit to single exponential decay with $\tau=13 \mathrm{~ns}$.

defect-related emission at low temperatures. The emission bands in the UV part of the spectrum are stronger in the fast time window, indicating that these are the transitions from the lowest $f-d$ state of $\operatorname{Pr}^{3+}$ which will have a fast (ns) decay time. The bands have been assigned to transitions from the lowest $f d$ state to the ${ }^{3} \mathrm{H}_{4}$, ${ }^{3} \mathrm{H}_{5},{ }^{3} \mathrm{~F}_{3}$ and ${ }^{1} \mathrm{G}_{4} 4 f$ states of $\mathrm{Pr}^{3+}$ for the bands located at 250, 263, 300 and $328 \mathrm{~nm}$, respectively. The separation between the bands agrees well with the known energy differences between the ${ }^{3} \mathrm{H}_{4}$, ${ }^{3} \mathrm{H}_{5},{ }^{3} \mathrm{~F}_{3}$ and ${ }^{1} \mathrm{G}_{4}$ states.

Under excitonic excitation at $165 \mathrm{~nm}$ (Fig. 6B), the emission spectrum consists of three broad bands in the range $210 \mathrm{~nm}$ to about $550 \mathrm{~nm}$. The maxima of the bands are located around $260 \mathrm{~nm}$ (with a small shoulder like feature at $237 \mathrm{~nm}$ ), $300 \mathrm{~nm}$ and at $390 \mathrm{~nm}$ (with a tail extending to about $550 \mathrm{~nm}$ ). The bands are stronger in the slow time window, indicating a long luminescence lifetime although some fast emission is observed between 240 and $320 \mathrm{~nm}$. These UV emission bands may have a contribution from $\mathrm{Pr}^{3+} d-f$ emission. Feeding of the $f d$ state through the excitonic state may also result in a longer lifetime for delayed $d-f$ emission. The fact that the emission bands are broader than under direct excitation may be caused by broadening resulting from differently charge compensated $\mathrm{Pr}^{3+}$ sites, which are excited non-selectively under excitonic excitation. However, also defect related emission from the $\mathrm{SrCl}_{2}$ host lattice has been observed in the spectral region around $300 \mathrm{~nm}$. Due to the strong overlap it is hard to assign the UV emission bands to $d-f$ or host lattice emissions. The strong emission band around $390 \mathrm{~nm}$ is assigned to defect emission. Its position is consistent with the bands observed in the emission spectrum of the undoped $\mathrm{SrCl}_{2}$ host lattice under ionizing radiation excitation, presented in Fig. 1.

The lifetime of the praseodymium $d-f$ emission in strontium chloride has been measured under a direct $f$ - $d$ excitation at $8 \mathrm{~K}$ for a sample activated with $0.5 \% \mathrm{Pr}^{3+}$. The decay curve is presented in Fig. 7. The decay is single exponential, with a decay time of 13 ns. This is much shorter than the decay times measured for $\mathrm{Ce}^{3+}$ emission and is promising for application as a scintillator material.

The emission spectra of $\mathrm{Pr}^{3+}$ in $\mathrm{BaCl}_{2}$ (at $8 \mathrm{~K}$ ) are shown in Fig. 8 for two different excitation wavelengths, $168 \mathrm{~nm}$ (excitonic) and $230 \mathrm{~nm}$ ( $f$ - $d$ excitation). Under a direct $f$ - $d$ excitation the emission spectrum consists of two sets of emission bands as shown in Fig. 8A. The emissions in the UV part of the spectrum are located at around 260, 288 and $315 \mathrm{~nm}$. These emission bands can be observed in the fast time window, indicating that they are related to the transitions from the lowest $f d$ state to the $4 f^{2}$ levels, ${ }^{3} \mathrm{H}_{4},{ }^{3} \mathrm{H}_{6},{ }^{3} \mathrm{~F}_{4}$, respectively. The $f d \rightarrow{ }^{3} \mathrm{H}_{5}$ is not observed as a separate band, probably because it overlaps with the $f d \rightarrow{ }^{3} \mathrm{H}_{4}$ transition which is close in energy. It should be noted that the emission band that has been assigned to the $f d \rightarrow{ }^{3} \mathrm{~F}_{4}$ transition overlaps with a slower decaying band around $310 \mathrm{~nm}$. This indicates that there is an emission of different nature present at that wavelength that can also be excited at $230 \mathrm{~nm}$, probably a host lattice related emission band. The emission bands between $350 \mathrm{~nm}$ and $500 \mathrm{~nm}$ are assigned to the defect related emissions. The small peak at $490 \mathrm{~nm}$ is probably ${ }^{3} \mathrm{P}_{0} \rightarrow{ }^{3} \mathrm{H}_{4}$ emission of $\mathrm{Pr}^{3+}$.

The emission spectrum of $\mathrm{BaCl}_{2}: \mathrm{Pr}^{3+} 2 \%$ under excitonic excitation at $168 \mathrm{~nm}$ (Fig. 8B) shows a very broad band from about 240 to $600 \mathrm{~nm}$, peaking at $390 \mathrm{~nm}$. This band is assigned to the host lattice emission, as the one observed in the literature for $\mathrm{BaCl}_{2}$ [22], similar to the band observed in $\mathrm{SrCl}_{2}$. There is also a sharper spectral feature present at about $490 \mathrm{~nm}$ which is assigned to the ${ }^{3} \mathrm{P}_{0} \rightarrow{ }^{3} \mathrm{H}_{4}$ emission. The presence of this emission demonstrates that there is energy transfer from the excitonic state to the $4 f$ levels of praseodymium. However, no transition from the lowest $f-d$ state has been observed in the region between 250 and $300 \mathrm{~nm}$, contrary to observation for $\mathrm{SrCl}_{2}: \mathrm{Pr}^{3+}$ where $d-f$ emission from $\mathrm{Pr}^{3+}$ was observed after excitonic excitation, albeit weak. This indicates that there is no energy transfer from the host lattice excitonic state to the $f d$ state of $\mathrm{Pr}^{3+}$ in $\mathrm{BaCl}_{2}: \mathrm{Pr}^{3+}$. An interesting spectral feature can be observed around $450 \mathrm{~nm}$. The peculiar dip in the host lattice emission spectrum is a clear signature of radiative energy transfer to the praseodymium ${ }^{3} \mathrm{P}_{2}$ level. This will be followed by emission from the ${ }^{3} \mathrm{P}_{0}$ level to the ${ }^{3} \mathrm{H}_{4}$ ground state. In addition to radiative energy transfer there may also be non-radiative transfer. The praseodymium $d-f$ emission lifetime in barium chloride has been measured at $8 \mathrm{~K}$ under a direct $f d$ excitation for the $2 \%$ doped sample. The luminescence decay curve is shown in Fig. 9. The decay curve has a non-exponential initial part followed by a more exponential tail. The tail has been fit to a single exponential which results in a decay time of $10 \mathrm{~ns}$. The non-exponential initial part can be explained by cross-relaxation quenching for neighboring $\mathrm{Pr}^{3+}$ ions. At $2 \%$ of $\mathrm{Pr}^{3+}$ it is known that cross-relaxation starts to depopulate the $f d$ excited state. The tail represents the decay for isolated $\mathrm{Pr}^{3+}$ ions and the fit gives a reliable estimate of the radiative decay rate of the $f d$ state. Some non-exponentiality may also be introduced by the presence of multiple sites with different local charge compensation. The 10 ns lifetime is among the shortest radiative lifetimes that have been reported for $\operatorname{Pr}^{3+} d-f$ emission. For example, the radiative lifetime for the $d-f$ emission from $\mathrm{Pr}^{3+}$ in the

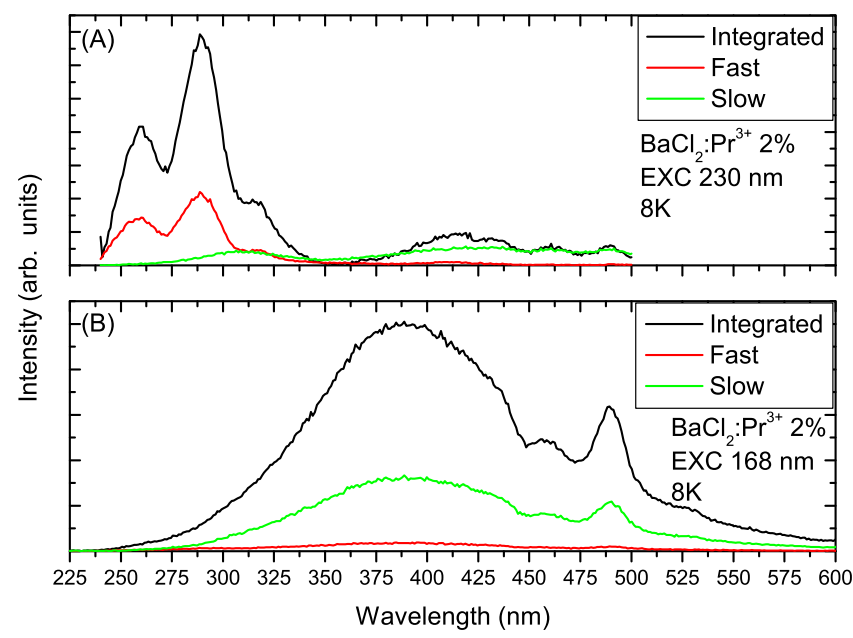

Fig. 8. Emission spectra of $\mathrm{BaCl}_{2}: \mathrm{Pr}^{3+} 2 \%$ for excitation into the host lattice at $168 \mathrm{~nm}$ (A) and direct excitation into the $f d$ band of $\mathrm{Pr}^{3+}$ at $230 \mathrm{~nm}$ (B). The spectra were recorded in different time windows (fast, slow and integrated, see also Section 2.2) at $8 \mathrm{~K}$. 


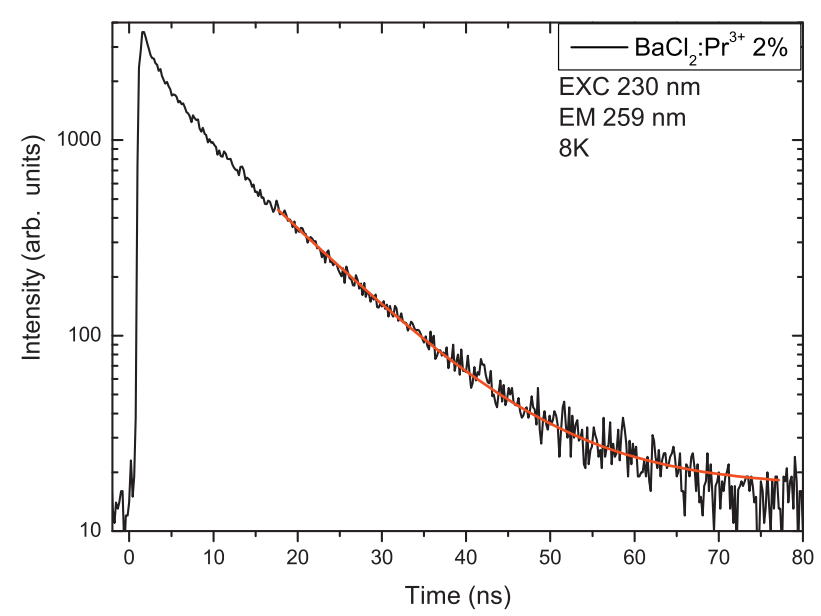

Fig. 9. Luminescence decay curve of the $\operatorname{Pr}^{3+} d-f$ emission ( $259 \mathrm{~nm}$ ) in $\mathrm{BaCl}_{2}: \mathrm{Pr}^{3+} 2 \%$ under direct excitation in the $f d$ band $(230 \mathrm{~nm})$ at $8 \mathrm{~K}$. The drawn line corresponds to a fit to single exponential decay for the tail of the curve with $\tau=10 \mathrm{~ns}$.

scintillator material LuAG: $\operatorname{Pr}$ is $22 \mathrm{~ns}$. The faster radiative decay rate in $\mathrm{BaCl}_{2}$ is explained by the shorter emission wavelength $(260 \mathrm{~nm})$ and the higher refractive index of the material. The short decay time is favorable for application in PET scanners where a shorter radiative lifetime contributes to a better image quality.

\subsection{4. $\mathrm{Nd}^{3+}$ doped $\mathrm{SrCl}_{2}$ and $\mathrm{BaCl}_{2}$}

The lowest energy $f d$ level for $\mathrm{Nd}^{3+}$ in $\mathrm{SrCl}_{2}$ is expected to be located around $185 \mathrm{~nm}$ based on the position of the $f d$ level for $\mathrm{Ce}^{3+}$ in $\mathrm{SrCl}_{2}$ and the shift of $22,720 \mathrm{~cm}^{-1}$ to higher energy predicted by the Dorenbos relation [3]. The emission spectrum of neodymium doped strontium chloride only shows slow $f$ - $f$ emission, even when it is excited directly into the $f d$ states $(185 \mathrm{~nm})$.

For $\mathrm{BaCl}_{2}: \mathrm{Nd}^{3+}$ the lowest $f d$ state of $\mathrm{Nd}^{3+}$ is expected around $189 \mathrm{~nm}$. Just as for $\mathrm{SrCl}_{2}: \mathrm{Nd}^{3+}$, no $d-f$ luminescence is observed, even under a direct $f-d$ excitation. Instead, there is feeding of the $4 f^{3}$ states of neodymium and only the slow intraconfigurational emission could be observed.

A possible explanation for the lack of $\mathrm{Nd} d-f$ emission in both $\mathrm{SrCl}_{2}$ and $\mathrm{BaCl}_{2}$ is that the lowest energy $f d$ band of $\mathrm{Nd}$ in these host lattices is at higher energies than host lattice excited states, leading to energy transfer from $f d$ states to defect states, which in turn populate high energy $4 f^{3}$ neodymium levels through energy transfer. It also cannot be excluded that the $f d$ states of $\mathrm{Nd}^{3+}$ are located close to or in the conduction band of $\mathrm{SrCl}_{2}$ and $\mathrm{BaCl}_{2}$. In this case excitation in the $f$ - $d$ state results in an impurity trapped exciton state that can relax by feeding the lower lying $4 f^{3}$ levels of $\mathrm{Nd}^{3+}$, leading to the slow $f-f$ emission.

\subsection{Radioluminescence}

Radioluminescence is an important tool in the investigation of potential scintillator materials. It allows to study the luminescence properties under X-ray excitation. Under high energy excitation the efficiency of energy transfer from the host lattice to the activator ion can be investigated along with the kinetics. Also the radiation stability can be evaluated for excitation with ionizing radiation. Finally, it allows for an evaluation of the overall scintillation efficiency and of (undesired) afterglow properties.

\subsection{1. $\mathrm{Ce}^{3+}$ doped $\mathrm{SrCl}_{2}$ and $\mathrm{BaCl}_{2}$}

Under ionizing radiation the $\mathrm{Ce}^{3+}$ emission for $\mathrm{SrCl}_{2}$ :Ce consists of two broad bands that are consistent in shape and spectral location with the spectra observed under UV excitation (see Fig. 2, red curve). This proves that the emission comes from $\mathrm{Ce}^{3+}$ in $\mathrm{SrCl}_{2}$. The bands are located at $360 \mathrm{~nm}$ and $384 \mathrm{~nm}$. Upon ending X-ray excitation afterglow was present and it could be measured (for the $0.5 \%$ doped sample) for up to about 8 min after removal of the excitation source. The recorded afterglow decay is presented in the inset of Fig. 2. The afterglow is related to the slow release of trapped charge carriers generated under X-ray excitation. Recombination of thermally released charge carriers on or near the $\mathrm{Ce}^{3+}$ ions results in $\mathrm{Ce}^{3+}$ emission. Traps for free charge carriers may pre-exist in the material but the formation of traps can also be induced by the high energy irradiation.

A peculiar change in the relative intensities of the two $\mathrm{Ce}^{3+}$ emission bands was observed during irradiation. Both bands become weaker but the decrease in intensity of the longer wavelength band is much stronger. Probably this is due to the formation of color centers which absorb more strongly at the wavelength of the longer wavelength emission band. After prolonged irradiation the sample turns grayish indicating that color centers are formed.

The scintillation efficiency increased when the $\mathrm{Ce}^{3+}$ concentration was increased from $0.5 \%$ to $2 \%$. The increase, based on the long wavelength band at $384 \mathrm{~nm}$, is of about $40 \%$ for the $2 \%$ doped sample, compared to the $0.5 \%$ one. This is due to the larger number of $\mathrm{Ce}^{3+}$ ions available for excitation. By increasing the concentration of the activator ions, the probability of the excitation energy being captured by them increases. Otherwise the energy would have been lost through competing channels (e.g. recombination at trap sites).

$\mathrm{Ce}^{3+}$ emission in $\mathrm{BaCl}_{2}$ has also been studied under ionizing radiation excitation. The emission spectrum consists of one broad band (red curve in Fig. 4), located around $358 \mathrm{~nm}$. The position is similar to that observed under UV excitation but the characteristic doublet structure of $\mathrm{Ce}^{3+}$ emission has disappeared. The spectrum is also different from previous reports in the literature [10-13], where two emission bands have been observed under ionizing radiation excitation. Selling et al. have also observed a weak band around $300 \mathrm{~nm}$ that they have ascribed to the STE emission in barium chloride [12,23]. No such emission has been observed in the present study. The reasons for such a discrepancy are not clear. The single broad emission band around $358 \mathrm{~nm}$ has been observed for all samples, with $\mathrm{Ce}^{3+}$ concentrations of $0.1 \%, 0.5 \%$ and $2 \%$. The origin of the change in the emission spectrum may be radiation induced defects. The softer $\mathrm{BaCl}_{2}$ is more prone to the creation of defects than $\mathrm{SrCl}_{2}$. Due to defect formation, the local environment of the $\mathrm{Ce}^{3+}$ ions changes and the disorder causes broadening of the $\mathrm{Ce}^{3+}$ emission bands, and therefore the $2000 \mathrm{~cm}^{-1}$ splitting can no longer be observed. This observation is in contrast to the results presented by Selling et al. [12], although it should be pointed out that in the cited reference the authors have used a different excitation source for X-rays. It is possible that the dose of the radiation was different in their case, which would lead to the observed difference, but this aspect has not been further investigated.

The afterglow of the $\mathrm{Ce}^{3+}$ emission in $\mathrm{BaCl}_{2}$ under X-ray excitation has been measured for the $0.5 \%$ doped sample. The emission could be detected for up to $6 \mathrm{~min}$ after the excitation has been stopped. The resulting afterglow decay is presented in the inset of Fig. 4. Just as in case of $\mathrm{SrCl}_{2}: \mathrm{Ce}^{3+}$ the afterglow is assigned to the slow thermally activated release of trapped charge carriers.

The highest scintillation intensity has been observed for the $2 \%$ doped sample and the intensity has increased in the concentration series from $0.1 \%$ to $2 \%$. The increase when going from the $0.1 \%$ doped sample to the $0.5 \%$ doped one is of about $18 \%$. When the concentration has been increased to $2 \%$, the increase in the scintillation efficiency compared to the $0.1 \%$ doped sample has been about $62 \%$. Just as in the case of $\mathrm{SrCl}_{2}: \mathrm{Ce}^{3+}$ this observation is explained in the terms of a greater number of activator ions available for excitation by the created electron-hole pairs (which number for a specific material and specific excitation is constant), leading to 
the increased probability that the excitation energy will reach the activators, leading to increased luminescence intensity.

\subsection{2. $\mathrm{Pr}^{3+}$ doped $\mathrm{SrCl}_{2}$ and $\mathrm{BaCl}_{2}$}

To investigate the efficiency of the fast $d-f$ emission of $\mathrm{Pr}^{3+}$ under $\mathrm{X}$-ray excitation, the emission spectrum of $\mathrm{SrCl}_{2}: \mathrm{Pr}^{3+}(0.5 \%$ and $2 \%)$ has been measured under X-ray excitation and is shown in Fig. 10. The spectrum consists of a broad band around $400 \mathrm{~nm}$ and two sets of sharp emission lines at about $490 \mathrm{~nm}$ and $600-650 \mathrm{~nm}$. The broad band emission is consistent with the previously observed host lattice emission of $\mathrm{SrCl}_{2}$. The sharp lines are assigned to the $\operatorname{Pr}^{3+} f-f$ emission. No $\operatorname{Pr}^{3+} d-f$ emission is observed. This indicates that the energy transfer from the electron-hole pairs created by the $\mathrm{X}$-rays to the $f d$ state of $\mathrm{Pr}^{3+}$ is inefficient. This is consistent with the VUV luminescence measurement discussed in the previous section. The host lattice emission is too low in energy and there is no spectral overlap with the $f$ - $d$ excitation bands of $\mathrm{Pr}^{3+}$, which are situated at high energies. There is spectral overlap with the $4 f^{2}$ excitation lines and as a result, there is partial energy transfer to the $4 f^{2}$ levels $\left({ }^{3} \mathrm{P}_{\mathrm{J}}\right)$ of $\mathrm{Pr}^{3+}$, resulting in the $f-f$ emission lines. The presence of host lattice emission in the spectrum of the $0.5 \%$ doped sample shows that at this dopant concentration there is only partial energy transfer from the host lattice to $\mathrm{Pr}^{3+}$. To increase the energy transfer efficiency the $\mathrm{Pr}^{3+}$ concentration has been increased to $2 \%$. This strongly reduces host lattice emission intensity, showing that the energy transfer from the host lattice is indeed more efficient, but also the $\mathrm{Pr}^{3+}$ emission intensity decreases by a factor of about 7. This is explained by the cross-relaxation quenching of the $\mathrm{Pr}^{3+}$ emission, which is well known to occur at higher Pr concentrations and starts at $\operatorname{Pr}^{3+}$ concentrations as low as $0.5 \%$.

The $\mathrm{Pr}^{3+}$ emission in $\mathrm{BaCl}_{2}$ under ionizing radiation excitation has been investigated by measuring the X-ray excited emission spectra for three samples with $\operatorname{Pr}^{3+}$ concentrations of $0.1 \%, 0.5 \%$ and $2 \%$. The spectrum of the $0.5 \%$ doped sample is presented in Fig. 11 and shows line emission between $490-550 \mathrm{~nm}$ and $600-725 \mathrm{~nm}$ due to $\operatorname{Pr}^{3+} f-f$ emission. Just as for $\mathrm{SrCl}_{2}: \operatorname{Pr}$, no $d-f$ emission is observed showing that there is only energy transfer from the host lattice excited states to the $4 f^{2}$ states of $\operatorname{Pr}^{3+}$ and not to the $f d$ states. A very low intensity band is observed around $325 \mathrm{~nm}$. It is most likely that this weak emission band is STE emission in $\mathrm{BaCl}_{2}$, since its position is consistent with the one reported by Selling et al. [10]. There is only very weak host lattice emission in the sample with $0.5 \%$ Pr. The emission spectrum of the $0.1 \%$ doped $\mathrm{BaCl}_{2}$ sample (not shown) does show a strong host lattice emission band around

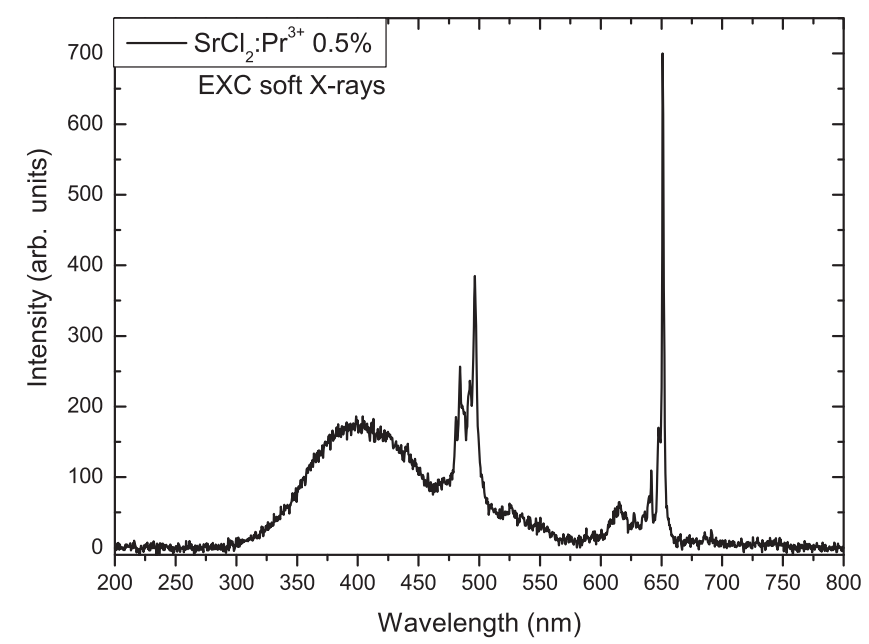

Fig. 10. Emission spectrum of $\mathrm{SrCl}_{2}: \mathrm{Pr}^{3+} 0.5 \%$ at $300 \mathrm{~K}$ under X-ray excitation (Cusource, operated at $40 \mathrm{kV}$ ).

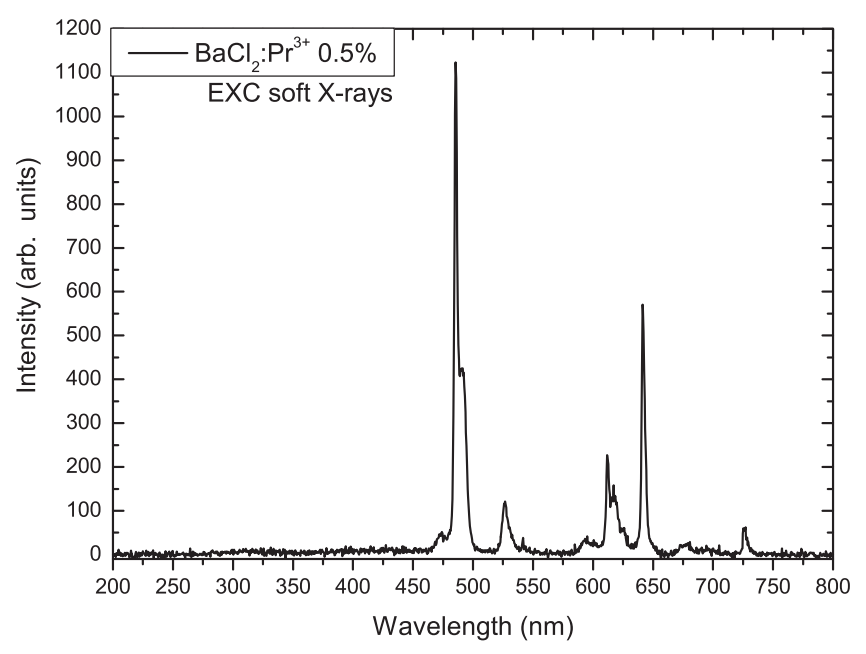

Fig. 11. Emission spectrum of $\mathrm{BaCl}_{2}: \mathrm{Pr}^{3+} 0.5 \%$ at $300 \mathrm{~K}$ under $\mathrm{X}$-ray excitation (Cusource, operated at $40 \mathrm{kV}$ ) at $300 \mathrm{~K}$.

$435 \mathrm{~nm}$. When the Pr content is increased to $2 \%$, the emission spectrum under X-ray irradiation is very similar to the one of the $0.5 \%$ doped material. The faint broad band emission is still present alongside the sharp line emissions of $\mathrm{Pr}^{3+}$. There is a decrease in the intensity of the praseodymium emission as compared to the $0.5 \%$ doped sample ( factor 1.6) due to cross-relaxation at the high $\mathrm{Pr}$ concentrations. The absence of $d-f$ emission in $\mathrm{BaCl}_{2}$ under excitation with X-rays is, just as for $\mathrm{SrCl}_{2}$ : $\mathrm{Pr}$, due to the lack of spectral overlap between the emission of the host lattice and the absorption of the $\operatorname{Pr} f d$ states which are situated at too high energies.

Comparison of the luminescence from $\mathrm{SrCl}_{2}: \mathrm{Pr}$ and $\mathrm{BaCl}_{2}: \mathrm{Pr}$ shows that the energy transfer from the host lattice to the $\mathrm{Pr}^{3+} 4 f^{2}$ states is more efficient in $\mathrm{BaCl}_{2}: \mathrm{Pr}$, leading to stronger $\mathrm{Pr}^{3+}$ emission in $\mathrm{BaCl}_{2}$ under $\mathrm{X}$-ray excitation. Secondly, the intensity ratio between the green ${ }^{3} \mathrm{P}_{0} \rightarrow{ }^{3} \mathrm{H}_{4}$ (around $500 \mathrm{~nm}$ ) and red ${ }^{3} \mathrm{P}_{0} \rightarrow{ }^{3} \mathrm{~F}_{J}$ (around 600-650 nm) $4 f$ emission is reversed for the two materials.

\section{Conclusions}

$\mathrm{SrCl}_{2}$ and $\mathrm{BaCl}_{2}$ host lattices have been doped with $\mathrm{Ce}^{3+}, \mathrm{Pr}^{3+}$ and $\mathrm{Nd}^{3+}$ to investigate the luminescence properties of the fast $d-f$ emission and the potential for application as a fast scintillation material. Efficient and fast $\mathrm{Ce}^{3+} d-f$ emission is observed in both $\mathrm{SrCl}_{2}$ and $\mathrm{BaCl}_{2}$ under direct $f$ - $d$ excitation, host lattice (VUV) excitation and $\mathrm{X}$-ray irradiation. The lifetime of the emission is $26 \mathrm{~ns}$ and $19 \mathrm{~ns}$ for $\mathrm{Ce}^{3+}$ in $\mathrm{SrCl}_{2}$ and $\mathrm{BaCl}_{2}$, respectively. After X-ray irradiation both $\mathrm{Ce}^{3+}$ activated compounds suffer from afterglow, which is most likely due to defect creation (e.g. color centers) by Xray irradiation. The barium chloride results are in good agreement with the ones presented by Selling et al. [12], with the position of the cerium emission band being the same and where the afterglow has also been observed. Only the doublet structure of the cerium emission is not present in the spectra presented in this work, which might be related to the dose of the ionizing radiation used.

For $\mathrm{Pr}^{3+}$ in $\mathrm{SrCl}_{2}$ and $\mathrm{BaCl}_{2}$ fast $d-f$ emission between 250 and $325 \mathrm{~nm}$ is observed under direct excitation into the $f d$ states. The emission and excitation spectra show an about $12,180 \mathrm{~cm}^{-1}$ and $12,610 \mathrm{~cm}^{-1}$ shift to higher energies of the lowest $f d$ state in comparison with the lowest $5 d$ state for $\mathrm{Ce}^{3+}$ in the same host lattice for strontium chloride and barium chloride, respectively, in relatively good agreement with the expected shift based on the Dorenbos formula of $12,240 \mathrm{~cm}^{-1}$. The lifetime of the $\mathrm{Pr}^{3+} d-f$ emission is about $13 \mathrm{~ns}$ in $\mathrm{SrCl}_{2}$ and $10 \mathrm{~ns}$ in $\mathrm{BaCl}_{2}$ which are among the fastest radiative decay times observed for $d-f$ emission from lanthanide ions. Under host lattice excitation and X-ray irradiation no $d-f$ emission 
is observed. Instead, slow host lattice related emission bands are observed along with $f-f$ emission. The absence of $d-f$ emission is explained by the high energy positions of the $f d$ excited states for $\mathrm{Pr}^{3+}$. The $f d$ states are at higher energies than the host lattice emissions of both $\mathrm{SrCl}_{2}$ and $\mathrm{BaCl}_{2}$ (situated between 300 and $500 \mathrm{~nm}$ ) and as a result there is no energy transfer from the host lattice to the $f d$ states of $\mathrm{Pr}^{3+}$ in $\mathrm{SrCl}_{2}$ and $\mathrm{BaCl}_{2}$. The absence of energy transfer from the host lattice to the $\mathrm{Pr}^{3+}$ fd states makes $\mathrm{SrCl}_{2}$ and $\mathrm{BaCl}_{2}$ doped with $\mathrm{Pr}^{3+}$ unsuitable for application as fast scintillator materials, in spite of the superior fast decay time in comparison to known $\mathrm{Ce}^{3+}$ and $\operatorname{Pr}^{3+}$ scintillator materials.

Emission spectra for $\mathrm{SrCl}_{2}$ and $\mathrm{BaCl}_{2}$ doped with $\mathrm{Nd}^{3+}$ under VUV and X-ray excitation show only intraconfigurational $4 f^{3}$ emission lines and host lattice related emission bands. No evidence for $d-f$ emission from $\mathrm{Nd}^{3+}$ was found. The absence for $d-f$ emission, even upon direct excitation in the $f d$ band, is explained by the high energy position of the $4 f^{2} 5 d$ levels of neodymium very close to or in the conduction band of $\mathrm{SrCl}_{2}$ and $\mathrm{BaCl}_{2}$.

\section{Acknowledgements}

The authors wish to acknowledge the financial support from the EU String project (NMP3-CT-2006-032636), as well as dr. A. Kotlov for help during the measurements at the Superlumi beam line at HASYLAB, DESY (Hamburg).

\section{References}

[1] W.W. Moses, Nucl. Instrum. Methods A 580 (2007) 919.
[2] G. Muehllehner, J.S. Karp, Phys. Med. Biol. 51 (2006) R117-R137.

[3] P. Dorenbos, J. Lumin. 91 (2000) 155.

[4] E. Zych, in: H. Singh Nalwa, L. Shea Rohwer (Eds.), Handbook of Luminescence Display Materials, and Devices, vol. 2, American Scientific Publishers, 2003, pp. 251-300.

[5] E.V.D. van Loef, P. Dorenbos, C.W.E. van Eijk, K.W. Krämer, H.U. Güdel, Nucl. Instrum. Methods A 486 (2002) 254.

[6] A. Bessiere, P. Dorenbos, C.W.E. van Eijk, K.W. Kramer, H.U. Güdel, C. de Mello Donegá, A. Meijerink, Nucl. Instrum. Methods A 537 (2005) 22.

[7] E.V.D. van Loef, P. Dorenbos, C.W.E. van Eijk, K.W. Kramer, H.U. Güdel, Nucl. Instrum. Methods A 496 (2003) 138.

[8] M.D. Birowosuto, P. Dorenbos, J.T.M. de Haas, C.W.E. van Eijk, K.W. Kramer, H.U. Güdel, J. Lumin. 118 (2006) 308.

[9] W. Drozdowski, A.J. Wojtowicz, Nucl. Instrum. Methods A 486 (2002) 412.

[10] J. Selling, S. Schweizer, M.D. Birowosuto, P. Dorenbos, IEEE Trans. Nucl. Sci. 55 (2008) 1183.

[11] J. Selling, Barium Halide Nanocrystals in Fluorozirconate based Glass Ceramics for Scintillation Application (PhD thesis, Universität Paderborn), 2007.

[12] J. Selling, S. Schweizer, M.D. Birowosuto, P. Dorenbos, J. Appl. Phys. 102 (2007) 074915.

[13] J. Selling, Ce-doped $\mathrm{BaCl}_{2}$ and fluorochlorozirconate glass-ceramic X-ray storage phosphors (MSc thesis, Universität Paderborn) 2004.

[14] O.T. Antonyak, I.V. Kityk, N.S. Pydzyrailo, Opt. Spectrosk. 63 (1987) 529.

[15] O.T. Antonyak, I.V. Kityk, N.S. Pydzyrailo, Opt. Spectrosk. 69 (1990) 606.

[16] W.M. Li, M. Leskelä, Mater. Lett. 28 (1996) 491.

[17] A. Zych, C. de Mello Donegá, A. Meijerink, J. Lumin. 129 (2009) 1535.

[18] PDF (Powder Diffraction File) \# 01-072-1537.

[19] PDF (Powder Diffraction File) \# 01-072-1388.

[20] G. Zimmerer, Nucl. Instrum. Methods A 308 (1991) 178

[21] Ch. Sugiura, Phys. Rev. B 9 (1974) 2679.

[22] K. Onodera, M. Koshimizu, K. Asai, Radiat. Phys. Chem. 78 (2009) 1031.

[23] J. Selling, M.D. Birowosuto, P. Dorenbos, S. Schweizer, J. Appl. Phys. 101 (2007) 034901 . 